Meta

Journal des tradlucteurs

Translators' Journal

\title{
Table des matières du volume 26
}

Volume 26, numéro 4, décembre 1981

URI : https://id.erudit.org/iderudit/003172ar

DOI : https://doi.org/10.7202/003172ar

Aller au sommaire du numéro

Éditeur(s)

Les Presses de l'Université de Montréal

ISSN

0026-0452 (imprimé)

1492-1421 (numérique)

Découvrir la revue

Citer cet article

(1981). Table des matières du volume 26. Meta, 26(4), 404-417.

https://doi.org/10.7202/003172ar d'utilisation que vous pouvez consulter en ligne.

https://apropos.erudit.org/fr/usagers/politique-dutilisation/ 


\section{TABLE DES MATIÈRES DU VOLUME 26}

\section{A. ARTICLES}

Andreyewski, Alexander: Translation: Aids, Robots, and Automation, 1, p. 57

Baudot, Jean: Un modèle de mini-banque de terminologie bilingue, 4, p. 315

Brace, Gerald : On Using On-Line Data-Bases for Help in Technical Translating, 1, p. 81

Brinkmann, Karl-Heinz: Machine Aids to Translation, 1, p. 67

Brunet, Jean-Paul : Le langage des «gendarmes » et des «voleurs», 2, p. 135

Bynner, John: Use of the Semantic Differential in Bilingualism Research and its Relevance to Translation, 3, p. 229

Chandioux, John: MÉTÉO : un système à l'épreuve du temps, 1, p. 18

Chevalier, Monique : La traductologie appliquée à la traduction automatique, 1, p. 35

Clas, André: Un modèle de mini-banque de terminologie bilingue, 4, p. 315

Fawcett, Peter: Teaching Translation Theory, 2, p. 141

Flamand, Jacques: Traducteur, philosophe, poète, qui est-il ?, 4, p. 350

Garretson, Deborah A. : A Psychological Approach to Consecutive Interpretation, 3, p. 244

Gémar, Jean-claude : Réflexions sur le langage du droit : problèmes de langue et de style, 4, p. 338

Goetschalckx, Jacques : Eurodicautom : problèmes résolus et problèmes posés, 1, p. 76

Gouadec, Daniel : Paramètres de l'évaluation des traductions, 2, p. 99

Gross, Maurice: Un modèle de mini-banque de terminologie bilingue, 4, p. 315

Guérard, Marie-France : MÉTÉO : un système à l'épreuve du temps, 1 , p. 18

Isabelle, Pierre : La traductologie appliquée à la traduction automatique, 1, p. 35

Kassai, Georges: Traduction et néologie, 2, p. 123

Kittredge, Richard : Foreword, 1, p. 5

Krollmann, Friedrich : Computer Aids to Translation, 1, p. 85

Labelle, François : La traductologie appliquée à la traduction automatique, 1, p. 25

Lainé, Claude : La traductologie appliquée à la traduction automatique, 1, p. 35

Melby, Alan K. : Translators and Machines - Can they Cooperate?, 1, p. 23

Pergnier, Maurice: Théorie linguistique et théorie de la traduction, 3, p. 255

Riva, Nina: Droit public et traduction, 3, p. 223

Romney, David: Use of the semantic Differential in Bilingualism Research and its Relevance to Translation, 3, p. 229

Schogt, Henri : Analyse sémantique immanente, référence et traduction, 2, p. 117

Schwab, Wallace: Traduction et informatique : perspective pour les années 80,1, p. 48

Spilka, Irène V. : Ambiguité et traduction, 4, p. 332

Van Hoof, Henri : La traduction scientifique : un phénomène récent?, 3, p. 215

Vauquois, Bernard : L'informatique au service de la traduction, 1, p. 8

\section{B. PROBLÈmES ET SOLUTIONS}

Aléong, Stanley: De vente à solde ou les dangers de l'hypercorrection, 2, p. 148

Baudot, Jean: Les fantômes en télévision, 4 , p. 376

Bennett, T.J.A. : Translating Colour Collocations, 3, p. 272 
Coppin, Mary : «Man» and Sexual Discrimination, 2, p. 168

Desgagné, Julie: Variations autour de la francisation des désignations de fonction, 3, p. 268.

Dubuc, Robert: Pour un vocabulaire bilingue de la production télévision, 3, p. 263

Grenier-Larocque, Suzanne: Or/Gold : étude comparative, 2, p. 172

Jammal, Amal : Les problèmes terminologiques de l'allergie, 4, p. 378

Jourdan, Christine: De vente à solde ou les dangers de l'hypercorrection, 2, p. 148

Kearney, Mary-Louise : La terminologie : méthode de définition des synonymes, 3, p. 284

Kromp, Richard: Néologie en marche... arrière ou avant?, 2, p. 175

Lethuillier, Jacques: Les étapes de la fabrication des circuits intégrés : termes en contexte, 4, p. 359

Lethuillier, Jacques: Le moteur Wankel : principe et terminologie, 4, p. 369

Lurquin, Georges: Mini, micro, nano, pico... ou la petitesse en informatique, 4, p. 381

Nakos-Aupetit, Dorothy: Traduction et terminologie : démarches comparées, 2, p. 159

Serré, Robert: Dictionnaire contextuel anglais-français de la chromatographie, 2, p. 281

\section{LES OUTILS DU TRADUCTEUR}

Serré, Robert: Ouvrages récents qui englobent un lexique ou un glossaire, 3, p. 290

Serré, Robert: Ouvrages récents qui englobent un lexique ou un glossaire, 4, p. 386

Spilka, Irène V. : Bibliographie, 2, p. 183

Spilka, Irène V.: Bibliographie, 3, p. 288

Spilka, Irène V. : Bibliographie, 4, p. 385

\section{COMPTES RENDUS}

Boutin-Quesnel, Rachel : Vocabulaire de l'habillement, 3, p. 302

Clas, André : Harrap's New Standard French and English Dictionary, 2, p. 195

Clas, André : Traduire sans trahir, 2, p. 291

Clas, André : Dictionnaire du bon français, 4 , p. 390

Dubuc, Robert: Dictionnaire de la langue québécoise, 2, p. 193

Elnitsky, Léo: International Seminar on Machine Translation, 2, p. 196

Hanna, Blake T.: Anthologie de la manière de traduire, 4, p. 387

Juhel, Denis : Bilinguisme et traduction au Canada : rôle sociolinguistique du traducteur, 3, p. 296

Larocque-DiVirgilio, Lise : Lectures et improvisations - incidences de la forme de l'énonciation sur la traduction simultanée (français-allemand), 3, p. 300

Larose, Robert: Traduire : théorème pour la traduction, 3, p. 292

Lethuillier, Jacques : Dictionnaire technique du pétrole, 2, p. 187

Muller, Gari : Le Jeu de la Feuillée, 4, p. 392

Pratt, Audrey: A Practical Guide to Bilingual Revision, 3, p. 298

Slote, Daniel: Guide de la traduction appliquée, 2, p. 190

Sparer, Michel : Bibliographie sélective du traducteur, 2, p. 185

Spilka, Irène V. : In Search of a Theory of Translation, 2, p. 191

Spilka, Irène V.: Lectures, 3, p. 295

\section{E. L'ACTUALITÉ}

Baudot, Alain: Table ronde I, 2, p. 204

Harris, Brian: Jean Herbert, 2, p. 208

Klein-Lataud, Christine : Table ronde II, 2, p. 208

Larocque-DiVirgilio, Lise : La traduction au magnétophone, 4, p. 398

Larose, Robert: Projets pédagogiques à l'U.Q.T.R., 3, p. 308

Quicheron, J.-B.: L'interprétation de congrès techniques ou l'art de l'impossible, 4, p. 396

Repa, Jindra: A Training Program for Court Interpreters, 4, p. 394

Seleskovitch, D. : Recherche universitaire et théorie interprétative de la traduction, 3, p. 304

Vanderveken, Alain: S.T.Q., 3, p. 309 


\section{INDEX DES MOTS ET DES SUJETS TRAITÉS DANS LE VOLUME 26}

à solder, 2, p. 153

accélérateur de particules, 4 , p. 363

acide fluorhydrique, 4 , p. 362

agrafer, 2, p. 140

AILA 81, 2, p. 207

allergie, 4 , p. 378

allergie gastro-intestinale, 4 , p. 378

allure, 3 , p. 285

alpaguer, 2, p. 140

altérer, 4 , p. 363

alvéole, 4 , p. 375

ambiguité discursive, 4 , p. 333

ambiguité et traduction, 4 , p. 332-337

ambiguïté linguistique, 4, p. 333

ambiguïté syntaxique, 3 , p. $261 ; 4$, p. 334

ambiguïtés d'analyse, 4, p. 330

amélioration de la race, 3 , p. 285

amélioration génétique, 3 , p. 285

amélioration zootechnique, 3, p. 285

amplitude d'écho, 4 , p. 377

ampoule, 4 , p. 361

analyse syntaxique, 4, p. 325

anaphylaxie, 4, p. 379

annonceur, 3, p. 265

aplanies, 4, p. 361

apport de chaleur, 4 , p. 375

arabismes en français, 2 , p. 128

arbre moteur, 4 , p. 375

arbre-excentrique, 4 , p. 375

arête, 4, p. 361

argilocinèse, 1, p. 82

argot, 2 , p. 135

argot de la production télévision, 3, p. 264

ARIANE-78, 1, p. 10

arquepincer, 2 , p. 140

arrêté royal, 3 , p. 227

ASLA, 2, p. 207

aspects contrastifs entre langue source et langue

but, 1, p. 14

attelage moteur, 4, p. 375

Austin, 2, p. 121

avoine, filer une, $2, \mathrm{p}, 140$

axiologie, 2, p. 119

azul, 2, p. 139

azurer, 3, p. 277

baigner dans le beurre, 2 , p. 138

bain de silicium, 4 , p. 361

baiser, se faire, 2 , p. 140

baisse exceptionnelle sur qqch., 2, p. 157

baisse sur qqch., 2, p. 157

banque de terminologie du Canada, 4, p. 316

banque de terminologie du Québec, 4 , p. 316

barbeau, 2, p. 138 barillet, 4 , p. 375

barreaux cylindriques, 4 , p. 361

bascule à Charlot, 2 , p. 137

belles infidèles, 2 , p. 126

bête à pain, 2, 138

bidochard, 2, p. 138

bifteck, 2, p. 138

bilinguisme et traduction au Canada, 3, p. 296

blanc-bleu, 2, p. 137

bleue, photocopie, 3, p. 274

bleuir, 3, p. 277

Bloomfield, L., 3, p. 257

bombardement, 4, p. 363

Bondarko, Alexander, 2, p. 121

bore, 4 , p. 360

bougie d'allumage, 4 , p. 375

bouleverser, 4, p. 363

bourre, 2 , p. 139

bourrique, 2, p. 139

brader, 2 , p. 157

braderie, 2, p. 157

brochet, 2, p. 138

brochet à la mie de pain, 2, p. 138

but de la traduction, 1, p. 14

cafard, le, 3 , p. 276

caild, 2 , p. 128

calcul des échos, 4, p. 377

calot, 3 , p. 302

calotte, 3, p. 302

camé, 2, p. 138

canaille, 2, p. 138

capacité de gonflement, 3 , p. 283

capsule de quartz, 4, p. 361

carburant, 2, p. 161

carter enveloppe, 4 , p. 375

catégories grammaticales, 2 , p. 119

cavalerie de la Maison du Roi, la, 3, p. 277

cave, le, 2 , p. 140

cavité, 4 , p. 375

CEDIJ, 1, p. 49

ceinturer, 2 , p. 140

cercler, 2 , p. 140

chambre d'injection, 3 , p. 282

chambre trochoïdale, 4 , p. 375

chariot à desserte, 2, p. 167

chariot à liqueurs, 2, p. 167

cheval, 2 , p. 138

chota, 2, p. 139

chromatographie de surface, 3 , p. 282

chromatographie, dictionnaire, 3, p. 281

chromatographie en phase vapeur, 3, p. 283

circuits intégrés monolithiques, 4 , p. 359

classes morpho-syntaxiques, 1, p. 11

cloche, 4 , p. 363 
comburant, 2 , p. $161 ; 4$, p. 375

combustible, 2 , p. 161

combustible liquide, 2 , p. 162

commande adaptative des feux de signalisation,

4 , p. 326

commande des signaux par le trafic, 4, p. 326

Commission des communautés europénnes,

Symposium, 2, p. 208

communicateur, 3, p. 266

communication entre délégué et interprète, 4, p. 397

compléments cognitifs, 3 , p. 260

complexité transparente, 4, p. 320

composants actifs, 4 , p. 360

composants passifs, 4 , p. 360

composition, procédés, 2 , p. 178

concentration du dopage, 4, p. 322

concepteur de circuit, 4, p. 361

condensateur, 2 , p. 161

condenseur, 2 , p. 161

conducteurs, 4, p. 359

conductivité électrique, 4 , p. 359

conre-champ, 3, p. 265

conseil constitutionnel, 3, p. 224

conseil d'État, 3, p. 224

conseil supérieur des Pays-Bas, 3, p. 224

constituants, 4, p. 325

contenant informatique, 4 , p. 319

contenu terminologique, 4 , p. 322

contigence et pertinence, 2 , p. 104

continuité, 3 , p. 265

contre-balayage, 3 , p. 281

contre-plongée, 3 , p. 265

coordination des signaux, 4 , p. 319

cour de cassation, 3, p. 224

créneau, 3 , p. 265

creuset, 4 , p. 361,363

croissance, 4 , p. 361

dauphin, 2, p. 138

De SAUSSURE, 2, p. 118

De Tende, G., 2, p. 125

décharge gazeuse, 4, p. 365

découper en tranches, 4 , p. 361

déflecteur, 2, p. 175

défoncé, 2, p. 138

dégriffé, 2, p. 157

délestage, 4, p. 329

densité des atomes dopants, 4, p. 322

dépôt par voie gazeuse, 4 , p. 363

dépôts de couches minces, 4, p. 363

dépression, 4 , p. 375

dermite atopique, 4 , p. 378

dermite atopique, 4 , p. 379

déshabillé, un, 2, p. 139

désignations de fonction, francisation des, 3 , p. 268

desserte roulante, 2 , p. 166,167

détente, 4 , p. 375

détérioration due aux échos, 4, p. 377

Devereux, G., 2, p. 128 déviation recommandée, 4 , p. 329

Dicautom, 4, p. 315, 316

dictaphone, 1, p. 53

diffuseur, 3 , p. 267

diffusion frontale, 3 , p. 282

diffusion thermique, 4, p. 362

diodes solides, 4 , p. 360

discours juridique, 4 , p. 344

dispositif anti-éblouissant, 2, p. 175

diviseur d'effluent, 3, p. 282

domaine, 1 , p. 14

domaine de linéarité, 3 , p. 282

dopage, 4, p. 323,360

dos vert, 2 , p. 138

drogue, vocabulaire angl.-fr., 2, p. 138

droit civil, 4, p. 339

droit et justice, bibliographie, 2, p. 185

droit public et traduction, 3 , p. 215

droper une dose, 2, p. 138

Dubeux, A., 2, p. 126

dur, 2, p. 137

éclairées, 4, p. 362

écrivains et traducteurs, table ronde, 2, p. 204

égalisées, 4, p. 361

électronique et lexicographie, 2, p. 208

électrons de valence, 4 , p. 359

électrons périphériques, 4 , p. 359

emballer, 2, p. 140

embarquer, 2, p. 140

émission induite, 2 , p. 165

émission stimulée, 2, p. 165

emprunts linguistiques, 2, p. 127

en promotion, 2, p. 157

en solde, 2, p. 153

encapsulés, 4 , p. 365

enseignement de la traduction, 2, p. 205; 3, p. 293

enseignement des langues, 3, p. 293

ensemble bielle-manivelle, 4 , p. 375

épée, 2, p. 137

épidermolyse bulleuse toxique, 4 , p. 381

épingler, 2, p. 140

épouser la veuve, 2, p. 137

épreuve à l'histamine, 4, p. 380

épreuve de libération d'histamine, 4, p. 380

équation d'écho, 4, p. 377

équipage mobile, 4 , p. 375

espace mort, 4, p. 375

espace résiduel, 4, p. 375

euphémismes, 4, p. 336

EUROTRA, 1, p. 8

évaluation des traductions, paramètres, 2, p. 99

évaluation et révision, 2, p. 99

évaporation sous vide, 4 , p. 363

excentration, 4 , p. 375

excentricité, 4 , p. 375

excentrique, 4 , p. 375

exposition-vente, 2, p. 156

fabrication des circuits intégrés, 4, p. 359,364

Fabri, P., 2, p. 125 
fabriqué, être, 2, p. 140

faces du texte, 2, p. 109

fait aux pattes, être, 2, p. 139

fait aux prunes, être, 2, p. 139

fait, être, 2 , p. 140

faute de traduction, 2, p. 104

fautes de traduction, 3 , p. 227

faux amis, 4 , p. 346

fenêtres, 4 , p. 362

fermer la marche, 3, p. 285

fiche de terminologie, 2 , p. 164

fiche de traduction, $2, \mathrm{p} .163$

ficher dedans, 2 , p. 140

fidélité, 4 , p. 352

filière française, 2, p. 138

finement polie, 4 , p. 361

fins de séries, 2, p. 157

FIT, congrès de Varsovie, 3 , p. 310

fixer, se, 2, p. 138

flag, en, 2, p. 139

flanquer dedans, 2, p. 140

flasque, 4, p. 375

flic, 2, p. 139

flicaille, 2 , p. 138

flique à dard, 2, p. 139

fluide moteur, 4 , p. 375

Fonagy, I., 2, p. 128

fonction critique, 4, p. 352

fonction réflexive, 4, p. 352

fonctions syntaxiques, 1, p. 11

fondu enchaîné, 3, p. 265

fondus, 3 , p. 265

four à oxydation, 4, p. 361

fourrer dedans, 2 , p. 140

francisation des désignations de fonction, 3, p. 268

franco, 2, p. 137

François, Frédéric, 2, p. 121

Frei, Henri, 2, p. 118,128

frontière, 4 , p. 360

Gara, L., 2, p. 125

gauler, se faire, 2 , p. 140

gaz porteur, 3 , p. 282

gaz vecteur, 3 , p. 282

GéoRef, 1, p. 82

germe, 4 , p. 361

grain cristallin, 4 , p. 359

grains monocristallins, 4 , p. 360

grand solde de qqch., 2 , p. 153

grande baisse sur qqch., 2, p. 157

grande mise en vente, 2, p. 150

grande vente à bon marché, $2, \mathrm{p} .150$

grande vente à escompte, notre, 2, p. 150

grande vente annuelle à une réduction spéciale,

$$
2 \text {, p. } 150
$$

grande vente de liquidation, $2, \mathrm{p} .150$

grande vente d'été, 2, p. 152

grande vente par encan, 2, p. 150

grande vente sans réserve, 2, p. 150

grandes démarques, 2 , p. 157

grandes occasions dans qqch., 2, p. 157 granulocyte basophile, 4, p. 379

grille, 3, p. 265

gros plan, 3, p. 265

groupe d'études pour la traduction automatique, 1, p. 8

Guiraud, P., 2, p. 127

habillement, vocabulaire de l', 3, p. 302

habillé, un, 2, p. 139

hareng, 2, p. 138

herbe, 2 , p. 138

Herbert, Jean, 2, p. 208

hirondelle, 2, p. 139

Hjemslev, Louis, 2, p. 118

homme, 2, p. 137

Hongrie, réforme linguistique, 2, p. 123

horizontal, 3 , p. 265

hypersensibilité, 4 , p. 378

Illyès, G., 2, p. 129

implantation ionique, 4 , p. 362

impuretés, 4, p. 360

index, 4 , p. 323

indice de rétention d'eau, 3, p. 283

informatique au service de la traduction, l', 1 , p. $1-94$

INFOTERM, 2, p. 207

ingénieur du son, 3, p. 265

injecteur à boucle, 3 , p. 281

Institut de textile français, 1, p. 93

intelligence dans la structure, 4, p. 319

interaction, 1 , p. 17

interférence due aux échos, 4 , p. 377

interlude, 3 , p. 265

interprétation de congrès techniques, l', 4 , p. 396398

introduire, 4, p. 361

isolants, 4, p. 359

ITS, 1 , p. 23-27

Jakobson, Roman, 2, p. 121

jardin des refroidis, 2, p. 137

jonctions, 4 , p. 360

Julot casse-croûte, 2 , p. 138

JURIVOC, 1 , p. 49

Karatson, A., 2, p. 124

Kazinczy, 2, p. 123

Kosztolanyi, 2, p. 124

La Place, 2, p. 126

labrocyte, 4 , p. 379

lame, 2, p. 137

lames, 4 , p. 361

langage du droit, 4 , p. $338-349$

langue de spécialité, 4 , p. 340-341

langue populaire, 2 , p. 136

langue québécoise, dictionnaire de la, 2, p. 193 
laque photosensible, 4 , p. 362

Larwill, H., 2, p. 125

Le Tourneur, 2, p. 126

Lebègue, R, 2, p. 126

lectures et improvisations, 3 , p. 300

Lederer, M., 3, p. 244

Leeb, E, 2, p. 128

lexicographie et électronique, 2, p. 208

lexie complexe, 2 , p. 178

lexique et syntaxe, 2, p. 104

LEXIS, 4, p. 316

liaisons, 4 , p. 359

lignes discontinuées, 2 , p. 157

limite de porosité, 3 , p. 282

lingots, 4 , p. 361

liquidation, 2, p. 157

liquidation de stock après inventaire, 2, p. 152

liquidation du stock avant départ, 2 , p. 155

LOCDE, 4, p. 315

LOCFRA, 4, p. 315

LOCIT, 4 , p. 315

LOCNE, 4, p. 315

logiciel de création, 4 , p. 320

logiciel d'interrogation, 4, p. 320

lot de tranches, 4 , p. 361

lumière, 4 , p. 375

lumière d'admission, 4 , p. 375

lumière d'échappement, 4 , p. 375

machine cinétique, 4 , p. 375

maladie de Bannister, 4, p, 379

maladie de Kussmaul-Meier, 4, p. 379

maladie de Milton, 4, p. 379

maladie du sérum de Rich, 4 , p. 380

maladie sérique, 4 , p. 380

malfrat, 2, p. 137

maneton, 4 , p. 375

mangeur de blanc, 2, 138

maquereau, 2, p. 138

maquignon, 2 , p. 138

marchand de barbaque, 2, p. 138

marchand de viande, 2 , p. 138

marchandises de pacotille, 2, p. 155

marmite, 2 , p. 137

marron, se faire faire, 2 , p. 140

Martinet, André, 2, p. 119

Maslov, Jurij, 2, p. 121

masques, 4 , p. 362

mastocyte, 4, p. 379

mécanisme bielle-manivelle, 4 , p. 375

médiatique, 1 , p. 48

Meillet, 3, p. 227

Mel'cuk, Igor, 2, p. 121

merde, 2, p. 138

merlan, 2, p. 138

Meschonnic, H., 2, p. 125

mesure d'écho, 4, p. 377

MÉTÉO, système, 1, p. 18-22

meulage, 4 , p. 361

mhos, 4 , p. 359

micro, 4 , p. 381

microcarte, 4 , p. 383 microcircuit, 4 , p. 382

microcoupure, 4 , p. 383

micro-ordinateur, 4 , p. 383

microplaquettes, 4 , p. 361

microprocesseur, 4 , p. 383

microprogrammation, 4 , p. 383

milieu, 2, p. 137

mini, 4, p. 381

mini-banques, 4 , p. 316,318

mis au rebut, 4 , p. 365

mis sous boîtier, 4, p. 365

mitan, 2, p. 137

modèle de mini-banque de terminologie bilingue, un, 4, p. 315-331

mois de qqch., 2, p. 157

Moncourt, 2, p. 127

monocristal, 4 , p. 360

moteur à capsulisme, 4, p. 375

moteur à combustion interne, 4, p. 375

moteur à piston rotatif Wankel, 4, p. 375

moteur à pistons, 4 , p. 375

moteur rotatif, 4, p. 375

moteur thermique, 4 , p. 375

moteur volumétrique, 4 , p. 375

moteur Wankel, 4, p. 369

moteur Wankel birotor, 4 , p. 375

moteur Wankel monorotor, 4, p. 375

motif élémentaire, 4 , p. 360

Mounin, Georges, 2, p. 121; 3, p. 227

mouvement rectiligne alternatif, 4, p. 375

multifiliérisation, 2, p. 176

nacelle, 4, p. 363

nanoseconde, 4 , p. 383

nature de la faute, 2, p. 104

néo-français, règles du, 2, p. 133

néologie-critères, 2 , p. 176

névrodermite généralisée, 4 , p. 379

Nida, E., 3, p. 258

niveau d'association de classes morphosyntaxiques, 1 , p. 10

niveau de construction des fonctions syntaxiques, 1 , p. 10

niveau de la construction logique, 1, p. 10

niveau de l'élément syntaxique de base affecté, 2, p. 108

niveau d'écho, 4 , p. 377

niveau d'interprétation des schémas arborescents, 1 , p. 10-14

nomenclature, 4 , p. 341

NORMATERM, 4, p. 316

objet du droit, 4 , p. 343

occasion, 2, p. 152

occasions, 2, p. 157

occasions exceptionnelles, 2, p. 157

adème de Quinke, 4, p. 379

offres spéciales, 2, p. 157

opérateur-disques, 3 , p. 267

ordonnance, 3 , p. 226

Oreme, N., 2, p. 125 
or/gold, étude comparative, 2, p. 172-174

$\mathrm{OSD}, 3$, p. 230

ouvertures, 4 , p. 362

Palmer, F.R., 2, p. 121

panoramique, 3, p. 265

paramètres de l'évaluation des traductions, 2 , p. 99

paramètres de traduction, 1, p. 14

paramètres situationnels du message, 3, p. 260

pas, 4, p. 360

PASCAL, 1, p. 82

passivation, 4 , p. 361

pastilles, 4, 361

pègre et police, vocabulaire, 2, p. 135

perdreau, 2, p. 139

Pergnier, M., 3, p. 244

périartérite noueuse, 4 , p. 379

pertinence et contingence, 2, p. 104

pescale, 2, p. 138

pétrole, dictionnaire du, 2, p. 187

philosophe et traducteur, 4 , p. 350

phonèmes, 2 , p. 118

phosphore, 4 , p. 360

photogravure, 4 , p. 362

pico, 4 , p. 381

pico-ordinateur, 4, p. 383

pipage, 2, p. 188

piper, se faire, 2 , p. 140

piquer, se faire, 2 , p. 140

piquouze, 2 , p. 138

piston crosse, 4 , p. 375

piston rotatif, 4, p. 375

plan, 4, p. 361

plan américain, 3, p. 265

plan d'ensemble, 3 , p. 265

PLATO(N), 3, p. 309

plongée, 3 , p. 265

poète et traducteur, 4 , p. 354

pointe, 4 , p. 375

pointeurs, 4 , p. 321,323

poisse, 2 , p. 138

poisson, 2 , p. 138

pollinose, 4 , p. 379

polycristallines, 4, p. 360

polymérisées, 4 , p. 362

pompe à vide, 4 , p. 363

porteurs de charge libres, 4 , p. 359

postédition, 1, p. 17

poulet, 2, p. 139

prédiction des échos, 4 , p. 377

prédiction des effets dus aux échos, 4, p. 377

prédiffusion, 4 , p. 362

préédition, 1 , p. 16

préparation des fiches, 4 , p. 324

présupposés de langue, 3 , p. 261

présupposés de parole, 3, p. 261

présupposition, 3, p. 261

principe de la recherche de descripteurs structuraux, 1, p. 9 principe de la séparation entre les données linguistiques et les programmes, 1, p. 10

prix chocs, 2, p. 157

prix coupés, 2, p. 157

prix de solde, 2 , p. 155

prix dégriffés, 2, p. 157

prix démarqués, 2 , p. 157

prix massacrés, 2 , p. 157

prix modérés, 2, p. 156

prix promotionnel, 2, p. 157

problème de réflexion, 4, p. 377

problème d'écho, 4 , p. 376

production, télévision, 3 , p. 263

profondeur de la pénétration, 4, p. 362

projeté, 4 , p. 363

promo, 2, p. 157

promotion de Noël, 2 , p. 157

promotion du mois, notre, 2 , p. 157

PS, 3, p. 224

puces, 4 , p. 361

puissance massique, 4, p. 375

pulvérisation cathodique, 4 , p. 363

pur, 2, p. 137

purisme esthétique, 2, p. 128

purisme naturel, 2 , p. 128

purpura capillarotoxique de Frank, 4, p. 379

purpura de Schönlein-Henoch, 4, p. 379

purpura rhumatoïde de Mathieu, 4, p. 379

pur-sang, industrie du, 3, p. 284

pylône écho, 4 , p. 377

quinzaine de qqch., 2, p. 157

raclette, 2, p. 139

radiotéléaste, 3 , p. 266

radiotélévision, 3, p. 267

rainurées, 4 , p. 365

ramasser, se faire, 2 , p. 140

rang-faute de traduction, 2, p. 105

rapport écho/signal, 4 , p. 377

rapport volumétrique de compression, 4 , p. 375

rayon des promotions, notre, 2 , p. 157

rayonnement ultraviolet, 4 , p. 362

réalisation de stock, 2 , p. 157

recherche ponctuelle, 2, p. 164

recherche thématique, 2, p. 164

recuire, 4 , p. 363

redistribution, 4 , p. 362

redonner, 4, p. 363

réforme linguistique hongroise, 2, p. 123

réfractaires, 4 , p. 364

réglo, 2 , p. 137

régul, 2 , p. 137

relations logiques, 1, p. 11

relativité des concepts, 4 , p. 343

remises exceptionnelles, 2 , p. 157

remises, grandes, 2 , p. 157

rentrer, 2 , p. 140

reproduction héliographique, 3 , p. 274

réseau cristallin, 4, p. 360 
résine, 4 , p. 362

résistivité, 4 , p. 359

restitution de la chaleur résiduelle, 4, p. 375

retard d'écho, 4, p. 377

rétrobalayage, 3 , p. 281

révision et évaluation, 2 , p. 99

Rey, A., 2, p. 128

rhume des foins, 4, p. 379

rideaux, 3, p. 265

rôle des constituants, 4 , p. 327

rôle-faute de traduction, 2, p. 105

rotor, 4 , p. 376

roussin, 2 , p. 139

Rozan, J.-F., 3, p. 245

russicismes en hongrois, 2 , p. 128

Saltesz, K., 2, p. 128

sang noble, 3 , p. 274

sauret, 2, p. 138

sauter, 2 , p. 140

schémas arborescents, 1, p. 10-14

se faire raccourcir, 2, p. 137

Searle, 2, p. 121

second coefficient du viriel, 3 , p. 282

secrétaire d'État, 3, p. 224

segment d'angle, 4 , p. 376

segment d'arrête, 4 , p. 376

segment de flanc, 4 , p. 376

segment périphérique, 4 , p. 376

segment radial, 4 , p. 376

Seleskovitch, D., 3, p. 245

semaine de qqch., 2, p. 157

sémantique et traduction, 2, p. 117

semiconducteurs de type n, 4 , p. 360

semiconducteurs de type p, 4, p. 360

semiconducteurs extrinsèques, 4 , p. 359

semiconducteurs intrinsèques, 4, p. 359

sensibilisation, 4 , p. 380

sensibilité, 4 , p. 378

sensitisation, 4 , p. 380

sérum allergène, $4, \mathrm{p} .380$

sérum allergisant, 4 , p. 380

servietter, 2, p. 140

SFIO, 3, p. 224

signifié, 2, p. 117

Shakespeare, traductions françaises, 2, p. 126

shooter, se, 2, p. 138

shooteuse, 2, p. 138

SIEMENS, 1, p. 67

signal écho, 4, p. 377

signalisation coordonnée, 4 , p. 319

signe linguistique, 2 , p. 117

signifiant, 2 , p. 117

signification du discours, 3, p. 262

signification en langue, 3, p. 262

silicium, 4 , p. 360

Simon, H., 2, p. 129

situation d'écho, 4 , p. 377

solde à l'entrepôt, 2, p. 152

solde après inventaire, 2, p. 152

solde d'anniversaire, 2 , p. 153

solde de clôture, 2, p. 152 solde de fermeture, 2 , p. 152

solde de places, 2, p. 153

solde de qqch., 2, p. 153

solde de rénovation, 2 , p. 152

solde d'éditeur, 2, p. 155

solde d'inventaire, 2, p. 152

solde et vente, 2 , p. 148

solde général, 2 , p. 152

solde petit-déjeuner, 2, p. 153

solder, 2, p. 153

soldes, 2 , p. 151

soldes avant départ, 2, p. 155

solide semi-conducteur, 4 , p. 359

sommet, 4, p. 376

sommets, 4 , p. 360

SOQUIJ, 1, p. 49

source chaude, 4 , p. 376

source froide, 4 , p. 376

SPD, 3, 224

speaker, 3, p. 265

speakerine, 3, p. 265

SRS-A, 4, p. 381

stages de traduction, 2, p. 205

stages en traduction, 3, p. 308

station, 3, p. 265

stator, 4 , p. 376

S.T.Q., assemblée générale, 3, p. 309

structure de réflexion, 4 , p. 377

structure des données, 4 , p. 321

structure écho, 4, p. 377

structure paradigmatique, 3 , p. 261

style indirect, 2 , p. 126

substance anaphylactique à réaction lente, 4 , p. 381

sucrer, 2, p. 140

super vente de tarifs, 2 , p. 151

syndrome de Holzknecht-Jacobson, 4, p. 379

syndrome de Lyell, 4, p. 381

synonymes, méthode de définition des, 3, p. 284

syntaxe et lexique, 2 , p. 104

système bielle-manivelle, 4 , p. 376

système d'évaluation positive des traductions, 2 ,

p. 111

SYSTRAN, système, 1, p. 8

table roulante, 2, p. 166

taperaux fafs, 2, p. 139

tarif promotionnel, 2, p. 157

tas, sur le, 2, p. 139

TAUM-AVIATION, 1, p. 37

TAUM, groupe, 1, p. 18-22

taux de compression, 4 , p. 376

taux de réponses moyen, 1, p. 78

TEAM, 4 , p. 316

technicien, 3, p. 265

téléastes, 3 , p. 266

temps d'exécution, 4 , p. 400

terminologie comparee, 2 , p. 159

terminologie et traduction, 2, p. 159

TERMIUM, 4, p. 316

TERMNET, 2, p. 207

texte scientifique, 2, p. 159 
texte technique, 2, p. 159

thème, 3, p. 293

théorie cinétique, 3, p. 282

théorie de la traduction, 3 , p. 255

théorie interprétative de la traduction, 3, p. 304

théorie linguistique, 3, p. 255

tisane, filer une, 2 , p. 140

titane, 4 , p. 364

Tolnai, Vilmos, 2, p. 123

tombo, 2, p. 139

torseur, 1, p. 81

toxico, 2, p. 138

traducteur juridique, formation du, 3, p. 225

traducteur, le philosophe et le poète, le, $4, \mathrm{p} .350$

traducteurs et écrivains, table ronde, 2, p. 204

traducteurs stagiaires, 3, p. 308

traduction au magnétophone, la, 4, p. 398-403

traduction automatique, 1, p. 8

traduction automatique, les méthodes, 1, p. 9

traduction automatique, limites de la, 1, p. 14

traduction automatisée assistée par le traducteur,

1 , p. 16

traduction biblique, 3, p. 291

traduction, enseignement de la, 2, p. 205

traduction et droit public, 3, p. 223

traduction et sémantique, 2, p. 117

traduction et terminologie, 2, p. 159

traduction humaine assistée par ordinateur, 1, p. 16

traduction juridique, 3, p. 223

traduction littéraire, table ronde, 2, p. 205

traduction par ordinateur, 1, p. 8

traduction scientifique, 3 , p. 215

traduction, stages en, 2 , p. 205

traduction technico-scientifique, 3, p. 215

traductologie, 1 , p. 36

traduire sans trahir, 3, p. 291

traduire, théorème pour la traduction, 3, p. 292

train, 3, p. 285

traînée, 3, p. 283

traitement multi-filière, 2 , p. 176

tranches, 4 , p. 361

travelling arrière, 3 , p. 265

travelling avant, 3, p. 265

très gros plan, 3, p. 265

trolley, 2 , p. 166

truand, 2, p. 137

tungstène, 4 , p. 364

turbine à gaz, 4 , p. 376

turbomachine réceptrice, 4 , p. 376

types d'erreurs, 4 , p. 401,402

typologie du texte, 1, p. 14

une de nos ventes les plus importantes, 2, p. 150

unité de traduction, 2, p. 160

unité terminologique, 2 , p. 160

unités de sens, 2 , p. 118

U.Q.T.R., projets pédagogiques, 3 , p. 308

urticaire chronique, 4 , p. 378

vache, 2, p. 139

valence, 4, p. 360 vapeur dopante, 4 , p. 362

vente à prix réduits, 2 , p. 151

vente à rabais, 2, p. 152

vente amiable, 2 , p. 156

vente annuelle de mi-hiver de qqch., 2, p. 154

vente au rabais, 2 , p. 151,152

vente, avoir qqch. en, 2 , p. 151

vente de clairance, 2 , p. 151

vente de feu, 2, p. 151

vente de fumée, 2 , p. 151

vente de garages, 2 , p. 151

vente de liquidation, 2 , p. 152

vente de Noël, 2, p. 151

vente de réclame, 2, p. 155

vente d'eau, 2, p. 151

vente d'écoulement de blanc à la verge, 2, p. 151

vente d'entrepôt, 2, p. 152

vente des tarifs, 2 , p. 152

vente d'occasion, 2 , p. 151

vente d'ouverture, 2, p. 152

vente en solde, 2, p. 152

vente et solde, 2, p. 148

vente exceptionnelle, 2, p. 152

vente extraordinaire, 2 , p. 150,152

vente promotionnelle, 2 , p. 157

vente réclame de rénovation, 2, p. 152

vente de soldes, 2, p. 152

vente spéciale, 2 , p. 156

vente spéciale de, 2, p. 150

vente, pas de, juste des bas prix, 2, p. 151

vente-réclame, 2 , p. 152

ventes à un cent, 2 , p. 151

verre transparent, 4 , p. 361

version dictée, 4 , p. 399

version manuscrite, 4 , p. 399

vertical, 3 , p. 265

veuve, la, 2, p. 137

vice-ministre, 3, p. 224

vilebrequin, 4 , p. 376

village, 2, p. 120

ville, 2 , p. 120

Vinay et Darbelnet, 3, p. 258

visière, 2 , p. 175

visions du monde, 3 , p. 257

vocabulaire de l'habillement, 3, p. 302

vocabulaire de soutien, 4 , p. 341

vocation du segment dans le texte, 2, p. 106

volet, 2 , p. 175

volet anti-éblouissant, 2, p. 176

volets, 3 , p. 265

volume nuisible, 4 , p. 376

voyou, 2, p. 137

vrai de vrai, le, 2 , p. 137

Whorf-Sapir, 3, p. 257

y aller du gadin, 2, p. 137

Zawadowski, Léo, 2, p. 119

Zuber, R., 2, p. 126 
INDEX OF WORDS AND TOPICS IN VOLUME 26

accuracy index, 1, p. 58

acid, 1 , p. $58 ; 2$, p. 138

active component, 4 , p. 365

address, 2 , p. 189

advertizing, de-sexing, 2 , p. 170

advisory diversion, 4,329

affective domain in translation, 2, p. 143

afterrunning, 2, p. 190

air-fuel ratio, 2 , p. 189

ALPAC report, 1, p. 25

alternative route, 4, p. 329

analysis, cluster, 3 , p. 241

analysis, statistical, 3, p. 241

angioedema, 4 , p. 379

angioneurotic edema, 4, p. 379

apex, 4,371

apex seal, 4 , p. 371

approach, decompositional, 3 , p. 248

argilocinesis, 1, p. 83

argilokinesis, 1, p. 83

artificial intelligence, 1, p. 58

artificial intelligence approach, 1 , p. 26

ass peddler, 2, p. 138

atopic dermatitis, 4 , p. 379

automobile emission control, 2, p. 189

balancing, 4 , p. 371

bargain, 2 , p. 151

bargain counter, 2, p. 155

bargain day, 2, p. 151

bargain prices, 2, p. 155

bargain sale, 2 , p. 151

Bar-Hillel, Y., 1, p. 25

basophil, 4, p. 379

batch, 4 , p. 365

batch processing system, 2 , p. 189

Besnier's prurigo, 4, 379

big shot, 2, p. 138

bilingual, coordinate, 3 , p. 229

black and white, 2, p. 139

blue boy, 2, p. 139

blue suit, 2 , p. 139

bond, 4, p. 365

boron, 4 , p. 365

boundary, 4 , p. 365

boys in blue, 2, p. 139

breed, improvement, 3, p. 285

broadcaster, 3, p. 267

broadcasting, 3, p. 267

brown sugar, 2 , p. 138

bulge, 4 , p. 37

bull, 2 , p. 139

burn, 2 , p. 139

bust, 2 , p. 139

butt peddler, 2, p. 138

bypass injector, 3, p. 28 cap, to, 4, p. 365

carrier gas, 3, p. 281

CAT system, 1, p. 86

cathodic sputtering, 4, p. 365

chili pimp, 2, p. 138

chip, 4, p. 365

chromatography, 3, p. 282

city, 2 , p. 120

clay, 1, p. 83

clay tectonics, 1, p. 83

clear, to, 2, p. 155

clearance space, 4 , p. 371

clipped, to be, 2, p. 140

coefficient, 2, p. 243

coefficient, second virial, 3 , p. 282

cognitive domain in translation, 2, p. 144

cold body, 4, p. 371

colligate, to, $2, \mathrm{p} .188$

colour collocations, 3 , p. 272

colour ellipsis, 3, p. 274

colour metaphors, 3, p. 273

comburent, 4 , p. 37

come to a rest, to, 4, p. 365

common law, 4 , p. 339

compression ratio, 4 , p. 372

compression space, 4 , p. 372

computer, digital, 2, p. 189

computer, hybrid, 2 , p. 189

computer output on microfilm, 1, p. 88

computer-aided translation system, 1, p. 86

condenser, 2, p. 161

conductivity, 4 , p. 365

conductor, 4, p. 365

contact lenses, 2 , p. 178

corner seal, 4 , p. 372

correlation, copheretic, 3 , p. 240

corrugate, to, 2, p. 188

court interpreters, 4 , p. 394

court interpreting, 4 , p. 394

crack salesman, 2 , p. 138

crankpin, 2, p. $189 ; 4$, p. 372

crankshaft, 2, p. $190 ; 4$, p. 372

crystal grain, 4 , p. 366

crystal lattice, 4 , p. 366

crystallite, 4 , p. 366

damp, to, 2, p. 188

data bases, on-line, 1 , p. 81

dead bang, 2, p. 139

dead space, 4 , p. 372

dermatitis, atopic, 4 , p. 379

diapirism, 1, p. 83

diastrophism, 1, p. 83

die, 4 , p. 366

dieseling, 2, p. 190

diffusion depth, 4 , p. 366

direct digital control, 2 , p. 189 
discard, 4, p. 366

displacement-type engine, 4 , p. 372

distributor, breaker type, 2 , p. 190

distributor, breakerless, 2, p. 190

dopant density, 4, p. 322

doping, 4, p. 323,366

doping concentration, 4 , p. 322

doping density, 4 , p. 322

doping level, 4, p. 322

drive-in diffusion step, 4 , p. 366

eccentric, 4 , p. 372

eccentricity, 4, p. 372

eccentric-rotor engine, 4 , p. 372

edema, angioneurotic, 4 , p. 379

electrical conductivity, 4, p. 366

eluent, 3 , p. 281

emission control, 2, p. 189

emission, stimulated, 2, p. 165

encapsulate, to, 4 , p. 366

end plate, 4, p. 372

epitrochoidal chamber, 4, p. 372

EURODICAUTOM system, 1, p. 67, 77

exclusion limit, 3 , p. 282

exhaust port, 4 , p. 372

expansion, 4 , p. 372

extrinsic conductor, 4 , p. 366

finder, 3 , p. 264

firing, 4 , p. 366

fish and shrimp, 2, p. 138

flat-bed chromatography, 3, p. 282

flipper. 2, p. 138

freak, 2, p. 138

french connection, 2, p. 138

fronting, 3, p. 282

fry, 2 , p. 137

fuel, 2, p. 161

fully automatic high quality translation, 1, p. 25

gaffer, 3, p. 264

gas discharge tube, 4 , p. 366

gas turbine engine, 4, p. 372

ghost computation, 4, p. 377

ghost delay, 4 , p. 377

ghost equation, 4 , p. 377

ghost impairment, 4, p. 377

ghost interference, 4, p. 377

ghost level, 4, p. 377

ghost magnitude, 4 , p. 377

ghost measurement, 4, p. 377

ghost prediction, 4, p. 377

ghost signal, 4, p. 377

ghost signal ratio, 4, p. 377

ghost situation, 4 , p. 377

ghost structure, 4 , p. 377

ghost tower, 4, p. 377

ghosting problem, 4 , p. 377

glass bell jar, 4, p. 366

gofer, 3 , p. 264 gold, 2, p. 172

goon, 2 , p. 137

grab, to, 2, p. 140

grass, 2 , p. 138

green room, 2, p. 137

growth, 4, p. 366

HAMT, 1, p. 5

harness bull, 2, p. 139

haul in, to, 2, p. 140

hearing aid, 2, p. 178

heat addition, 4 , p. 372

heat engine, 4, p. 372

heat input, 4, p. 372

heat rejection, 4, p. 372

heat resistant, 4, p. 366

high, to be, 2, p. 138

high vacuum, 4 , p. 366

histamine release test, 4 , p. 380

histamine test, 4 , p. 380

hole, 4, p. 366

home grown, 2, p. 138

homocytotropic antibody, 4 , p. 381

hood, 2, p. 137

hoodlum, 2, p. 137

horse, 2, p. 138

hot body, 4 , p. 372

hot chair, 2, p. 137

hot squat, 2, p. 137

hydrofluoric acid, 4, p. 366

hype, 2 , p. 138

ignition plug, 4 , p. 372

illuminate, to, 4 , p. 366

impurity, 4, p. 366

impurity semiconductor, 4 , p. 366

injection, 3, p. 282

inlet system, 3 , p. 282

insulator, 4, p. 366

intake port, 4, p. 372

intelligence, artificial, 3 , p. 247

interactive guidance, 1, p. 5

interactive translation system, 1, p. 23-27

internal combustion engine, 4, p. 372

international association of applied linguistics,

2, p. 207

international network for terminology, 2, p. 207

interpretation, consecutive, 3 , p. 244

interpreter's manual, 2, p. 208

intrinsic semiconductor, 4 , p. 366

inventory clearance, 2, p. 152

ion implantation, 4 , p. 367

job titles, 2, p. 168

joint, 2, p. 138

juicer, 3 , p. 264

jonction, 4, p. 367

junky, 2, p. 138

KWIC law, 1, p. 49 
language quality measurement system, 3 , p. 298

lattice, 4 , p. 367

lattice constant, 4 , p. 367

lattice spacing, 4 , p. 367

legit, 2 , p. 137

level, 2 , p. 137

LEXIS system, 1, p. 67

light-sensitive resin, 4 , p. 367

linear dynamic range, 3 , p. 282

linked traftic signal control, 4 , p. 319

logic, mathematical, 3 , p. 248

loop injector, 3 , p. 281

LSD, 2, p. 138

machine aided translation, 1, p. 5,57

machine aids to translation, 1 , p. 1-94

machine translation history, 1, p. 24

machine translation systems, 1, p. 5

mackerel, 2, p. 138

magnetic stripe, 3 , p. 264

magnetic tape exchange format, 1, p. 78

MAHT, 1, p. 5

man, 2, p. 137

man, in job titles, 2 , p. 168

mask, 4 , p. 367

mast cell, 4, p. 379

MAT, 1, p. 57

MATER format, 1, p. 70

material, 2, p. 188

meal ticket, 2, p. 138

meaning, affective, 3, p. 229

meaning, denotative, 3 , p. 229

meaning postulate, 3 , p. 249

melt, 4 , p. 367

memory in consecutive interpretation, 3, p. 246

mho, 4 , p. 367

micro, 4 , p. 381

microcarte, 4 , p. 383

microcolumn, 4 , p. 383

microcomputer, 4 , p. 383

microprocessor, 4, p. 383

$\operatorname{mini}, 4$, p. 381

mini-cassette, 4 , p. 382

minicomputer, 4 , p. 382

mobile charge carrier, 4 , p. 367

model home, 2, p. 178

monolithic integrated circuit, 4 , p. 367

moving parts, 4 , p. 373

MT systems, 1, p. 5

multithreading, 2, p. 176

nab, to, 2, p. 140

nail, to, 2 , p. 140

nano, 4 , p. 381

nanosecond, 4, p. 383

necktie, 2 , p. 137

nick, to, 2, p. 140

non-displacement type engine, 4 , p. 373

non-robotic translation, 1, p. 6,58

note-taking, consecutive, 3 , p. 245

n-type semiconductor, 4, p. 367 on-line data bases, 1, p. 81

outer electron, 4, p. 367

overdose, 2, p. 138

oxidizer, 4, p. 373

oxydation furnace, 4, p. 367

package, to, 4 , p. 367

pancake turner, 3 , p. 264

particle accelerator, 4 , p. 367

passivation, 4 , p. 367

passive component, 4 , p. 367

performance evaluation index, 1, p. 58

person, in job titles, 2, p. 170

phosphorus, 4 , p. 367

photoetching, 4, p. 367

photoresist, 4 , p. 367

pico, 4, p. 381

pig, 2, p. 139

pill party, 2, p. 137

pinch, to, 2, p. 140

piston and rod assembly, 4, p. 373

piston engine, 4 , p. 373

plain-clothes man, 2, p. 139

planar chromatography, 3, p. 282

plane chromatography, 3 , p. 282

planetary, 4 , p. 367

police raid, 2, p. 139

polycristalline, 4 , p. 367

polymerize, to, 4 , p. 368

popcorn pimp, 2, p. 138

port, 4 , p. 373

post-edition, 1, p. 5

pot, 2, p. 138

power-to-weight ratio, 4 , p. 373

predeposit step, 4 , p. 368

pre-edition, 1, p. 5

p-type semiconductor, 4 , p. 367

puking, 2, p. 188

pull in, to, 2 , p. 140

quartz boat, 4 , p. 368

R. c. engine, 4 , p. 373

reagin, 4 , p. 381

recess, 4 , p. 373

reciprocating engine, 4 , p. 373

reciprocating motion, 4 , p. 373

red eye, 2, p. 139

red-handed, 2, p. 139

regular, 2, p. 137

reliability index, 1, p. 58

remainder, 2 , p. 155

remainder line, 2, p. 155

resistivity, 4 , p. 368

retainer pin, 4 , p. 373

revision, a practical guide to bilingual, 3, p. 298

rhumatoid arthritis, 4, 381

right guy, 2 , p. 137

robotic translation, 1, p. 6,58

rotary combustion engine, 4 , p. 373

rotary engine, 4, p. 373 
rotating slider crank mechanism, 4, p. 373 rotor, 4 , p. 373

rotor housing, 4 , p. 373

rule of thumb in translation, 2, p. 142

run in, to, 2, p. 140

run-on, 2 , p. 190

sale, going-out-of-business, 2, p. 149

sale, anniversary, 2 , p. 149

sale, annual, 2, p. 150

sale, apartment, 2, p. 149

sale, back-to-school, 2, p. 150

sale, bankruptcy, 2, p. 148

sale, bargain, 2, p. 149

sale, Boxing day, 2, p. 150

sale, breakfast, 2, p. 150

sale, Christmas, 2, p. 150

sale, clearance, 2 , p. 149

sale, closing, 2 , p. 149

sale, closing down, 2, p. 152

sale, discount, 2, p. 149

sale, end-of-season, 2, p. 150

sale, fall, 2 , p. 150

sale, fire, 2, p. 149

sale, garage, 2, p. 149

sale goods, 2, p. 155

sale, half price, 2 , p. 149

sale, inventory, 2 , p. 149

sale, liquidation, 2, p. 149

sale, manager's, 2, p. 149

sale, one cent, 2, p. 149

sale, one dollar, 2, p. 149

sale, opening, 2, p. 149

sale, red tag, 2, p. 149

sale, renovation, 2 , p. 149,152

sale, rummage, 2 , p. 149

sale, sacrifice, 2 , p. 149

sale, sidewalk, 2, p. 149

sale, smoke, 2, p. 149

sale, spring, 2, p. 150

sale, storewide, 2, p. 149

sale, summer, 2, p. 150

sale, super-saver, 2 , p. 149

sale, surprise, 2, p. 149

sale, trans-Canada, 2, p. 149

sale, warehouse, 2, p. 149

sale, water, 2 , p. 149

sale, winter, 2 , p. 150

sales are on, 2, p. 155

salt tectonics, 1 , p. 83

sample home, 2, p. 178

scribe, to, 4 , p. 368

seed, 4 , p. 368

semantic differential in bilingualism, 3, p. 229

semiconductor, 4 , p. 368

sensitization, 4 , p. 380

serum sickness, 4 , p. 380

shale, 1, p. 83

shale diapirism, 1, p. 83

shale tectonics, 1, p. 83 shit, 2, p. 138

shoot, 2, p. 138

side housing, 4, p. 373

side plate, 4 , p. 373

side seal, 4 , p. 373

silcretes, 2 , p. 188

silicon, 4 , p. 368

silicon cylinder, 4 , p. 368

single crystal, 4 , p. 368

single-rotor Wankel engine, 4, p. 373

skull-cap, 3, p. 302

slab oil, 2 , p. 188

slice, 4, p. 368

slice, to, 4 , p. 368

slop in, to, 2, p. 140

slouched, to be, 2, p. 140

slow-reacting substance of anaphylaxis, 4, p. 381

smokey, 2, p. 139

sniffer, 2, p. 138

software, 2 , p. 178

solid-state diode, 4, p. 368

solvent regain, 3 , p. 283

spark plug, 4, p. 373

speech-acts, 2 , p. 121

speed, 2 , p. 138

splitter, effluent, 3, p. 282

square dealer, 2 , p. 137

square shooter, 2, p. 137

SRS-A, 4, p. 381

stick, 2, p. 138

stock taking sale, 2, p. 155

stoned, 2, p. 138

suck the pipe, 2, p. 137

sub-language approach, 1 , p. 26

supervisory control, 2, p. 189

sweat, to, 2, p. 140

swedish association of applied linguistics, 2, p. 207

tailing, 3 , p. 283

take in, to, 2, p. 140

tape, 3, p. 265

taping, 3, p. 265

tea trolley, 2, p. 166

tea wagon, 2, p. 166

teacart, 2, p. 166

TEAM system, 1, p. 67

tectonics, 1 , p. 83

terminological data elements, 2, p. 207

terminology data bank, 1, p. 61

theory, rate, 3, p. 282

thermal diffusion, 4 , p. 368

thin film deposit, 4, p. 368

thug, 2, p. 137

timing, 2, p. 188

tip, 4, p. 373

tire casing, 2, p. 189

TITUS project, 1, p. 93

town, 2 , p. 120

training program for court interpreters, 4 , p. 394 398 
translation teaching of, 2, p. 141

translation theory, 2, p. 141

translators/writers, 2 , p. 204

trolly, 2, p. 166

trunk piston, 4 , p. 375

tulsa, 1, p. 82

twistor, 1, p. 81

two-rotor Wankel engine, 4, p. 375

ultraviolet radiation, 4 , p. 368

underworld, 2, p. 137

unit cell, 4, p. 368

upset, to, 4 , p. 368

vacuum, 4 , p. 375

vacuum deposition, 4 , p. 368

vacuum pump, 4 , p. 368

valence, 4 , p. 368

valence electron, 4 , p. 368 valve injector, 3, p. 281

vapor-phase chromatography, 3 , p. 283

vehicle actuated signalization, 4 , p. 326

vehicle actuated traffic signals, 4, p. 326

vertex, 4 , p. 368

videocassette, 3 , p. 264

videotape, 3, p. 264

village, 2, p. 120

visor, $2, \mathrm{p}, 175$

wafer, 4 , p. 368

wage-price freeze, 2, p. 178

Wankel engine, 4, p. 375

water regain, 3, p. 283

white man, 2, p. 13 ?

whiz, 2, p. 137

window, 4 , p. 368

work over, to, 2 , p. 140

working agent, 4 , p. 375

writers/translators, 2, p. 204 